\title{
GASTRIC AND COLORECTAL METASTASES OF LOBULAR BREAST CARCINOMA: A CASE REPORT
}

\author{
David Buka ${ }^{1}$,Josef Dvořák ${ }^{2}$, Igor Richter ${ }^{3}$, Nikolov Dimitar Hadzi, Jiří Cyrany $^{5}$
}

\begin{abstract}
Department of Oncology and Radiotherapy, Charles University, Faculty of Medicine in Hradec Králové, University Hospital, Hradec Králové, Czech Republic ${ }^{1}$; Department of Oncology, First Faculty of Medicine, Charles University and Thomayer Hospital, Prague, Czech Republic ${ }^{2}$; Department of Oncology, Regional Hospital Liberec, Liberec, Czech Republic ${ }^{3}$; Department of Pathology, Charles University, Faculty of Medicine in Hradec Králové, University Hospital, Hradec Králové, Czech Republic ${ }^{4}$; 2nd Department of Internal Medicine - Gastroenterology, Charles University, Faculty of Medicine in Hradec Králové, University Hospital, Hradec Králové, Czech Republic ${ }^{5}$
\end{abstract}

Summary: Background: Occurrence of gastric metastasis as the first symptom of breast carcinoma with a long period of latency before presentation of the primary breast carcinoma is rare. Case Report: A patient with gastric metastasis as the first symptom of lobular breast carcinoma, treated by neoadjuvant preoperative chemoradiotherapy and total gastrectomy, with complete local control. Fourteen months after presentation of the gastric metastasis a primary lobular breast carcinoma was discovered, treated by radiotherapy, chemotherapy and hormonal treatment with complete local response. Twenty-three months after diagnosis of breast cancer multiple colorectal metastases from the breast cancer occurred, which were treated by chemotherapy and hormonal treatment. Eighty-six months after diagnosis of gastric metastasis the patient died due to progression of cancer. Conclusions: Metastases to gastrointestinal or gynaecological tracts are more likely in invasive lobular carcinoma than invasive ductal cancer. The pathologist should determine whether or not they check estrogen and progesterone receptor status not simply by signet ring cell morphology but also by consideration of clinic-pathological correlation of the patient, such as the presence of a past history of breast cancer, or the colorectal localization of poorly differentiated carcinoma, which may occur less frequently than in the stomach.

Keywords: Lobular breast carcinoma; Gastric metastasis from breast carcinoma; Multiple colorectal metastases from breast carcinoma

\section{Introduction}

McLemore et al. reported that cases of gastrointestinal metastasis from primary breast cancer are as rare as 73 cases among 12,001 cases. From these, only 24 cases with colorectal metastasis were recorded (1). The occurrence of gastric metastasis as the first symptom of breast carcinoma with a long period of latency before presentation of the primary breast carcinoma in this case report is even more infrequent. We report a patient with gastric metastasis as the first symptom of lobular breast carcinoma, who was treated by neoadjuvant preoperative chemoradiotherapy and total gastrectomy, with complete local control. Fourteen months after presentation of the gastric metastasis a primary lobular breast carcinoma was detected, and then treated by radiotherapy, chemotherapy and hormonal treatment with complete local response. Twenty-three months after diagnosis of breast cancer multiple colorectal metastases from the breast cancer occurred, which were treated by chemotherapy and hormonal treatment. Thirty-eight months after diagnosis of multiple colorectal metastases ascites occurred, and subsequently edema of the lower extremities and bi- lateral pleural effusion. Eighty-six months after diagnosis of gastric metastasis the patient died due to progression of cancer.

\section{Case report}

A 58-year-old woman was examined for abdominal pain and weight loss of $6 \mathrm{~kg}$. The biopsy from gastroscopy was assessed as a common dissociated gastric carcinoma with numerous signet ring cells (Figs. 1 and 2). Pretreatment stage was assessed as T2N0M0 according to endosonography, abdominal ultrasound, abdominal CT scan and chest X-ray, with normal pretreatment levels of CEA $3.39 \mu \mathrm{g} / \mathrm{l}$, CA 19-9: $18.45 \mathrm{U} / \mathrm{ml}$ and CA 72-4: $3.12 \mathrm{U} / \mathrm{ml}$. The patient was treated by neoadjuvant preoperative chemoradiotherapy which consisted of two 3-week cycles: 5-fluorouracil $300 \mathrm{mg}$ continuously days $1-21$, weekly cisplatin $40 \mathrm{mg}$ and weekly paclitaxel $90 \mathrm{mg}$. Concomitant with the second course of chemotherapy, radiotherapy of stomach and regional lymph nodes was administered with 30 Gy in 15 fractions from a linear accelerator. Radiotherapy was potentiated by ultrasound hyperthermia once weekly. Surgery was performed within 
5 weeks after completion of chemoradiotherapy. Total gastrectomy with concurrent cholecystectomy and splenectomy was performed. The histological examination of the resected stomach revealed coarse mucosa, which was densely infiltrated by the cells of dissociated gastric carcinoma with numerous signet ring cells. These cells penetrated through the muscularis mucosa and were dispersed throughout the wall, including the subserosa. Examined lymph nodes were without metastasis. Gallbladder and spleen were also without pathological changes. After surgery 6 cycles of adjuvant chemotherapy tegafur and calciumfolinat were administered.

Fourteen months after presentation of the gastric metastasis, lump in the right breast was detected during follow-up.

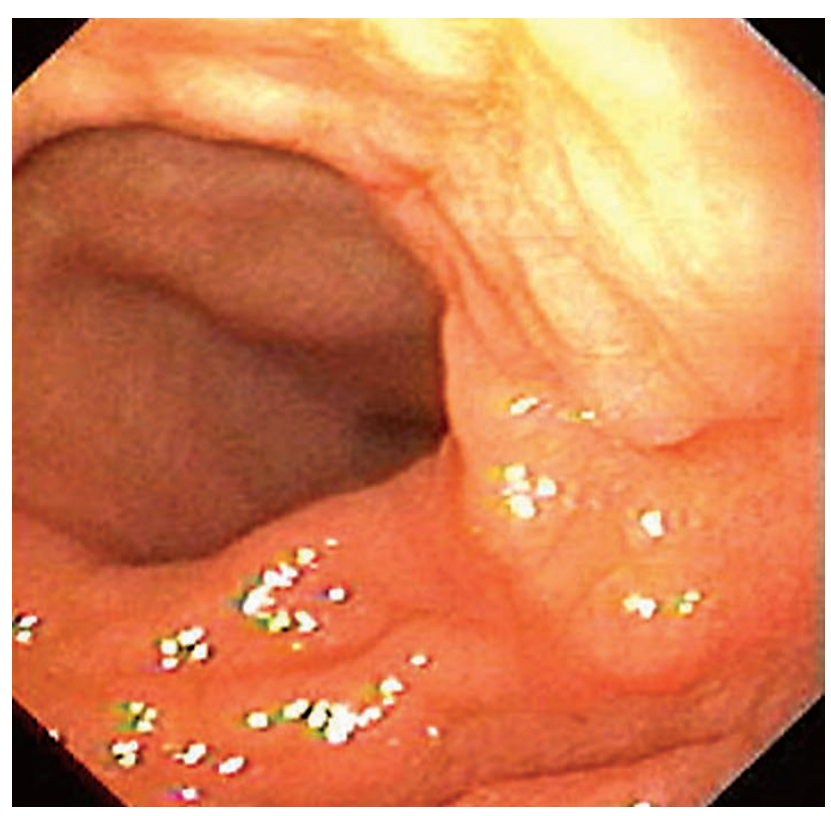

Fig. 1: Gastroscopy: There is a polypoid infiltration of the gastric mucosa in the dorsal wall in the region of the transition of the fundus and antrum of the stomach on the righthand side of the figure.

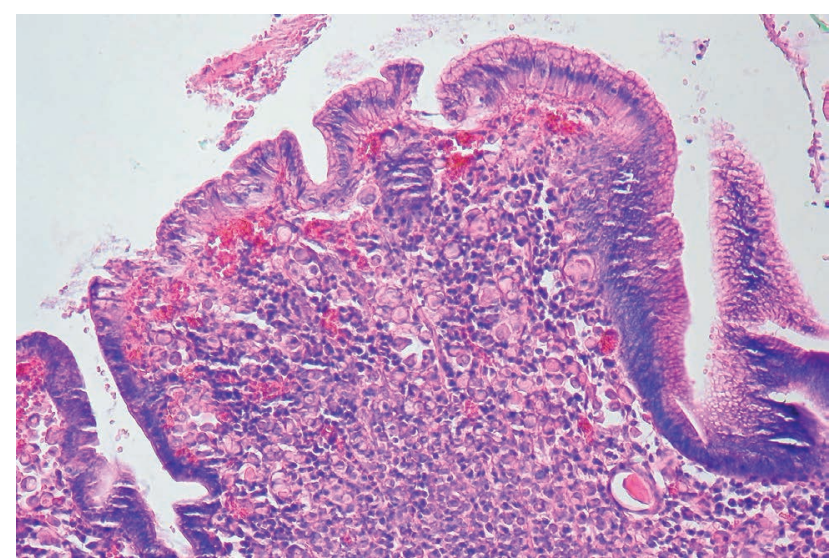

Fig. 2: Histological examination of biopsy from the stomach: coarse mucosa that is densely infiltrated by cells of a dissociated carcinoma with numerous signet ring cells.
Following mammography, this lump was evaluated as T3N0 breast carcinoma. Chest X-ray, abdominal ultrasound and bone scan did not indicate any distant metastases. Core biopsy demonstrated invasive lobular cancer, provisional grade 2, ER 6/8, PR 8/8 (modified Allred score) her 2 negative. Six cycles of chemotherapy in combination with docetaxel, epirubicin and cyclophosphamide were administered. After chemotherapy was started hormonal treatment with tamoxifen $(20 \mathrm{mg})$ once daily. After 6 cycles of chemotherapy, the control mammography showed no focal changes in the right breast or lymphadenopathy in the ipsilateral axilla or supraclavicular region. According to the multidisciplinary team the decision not to perform surgery was made. Radiotherapy was administered from a linear accelerator to the region of the right breast, the ipsilateral axillary and supraclavicular lymph nodes.

The patient was in complete remission for 23 months. Then the first subjective problems started: flatulence, belching, and diarrhea, without spasms, pain, or weight loss. Twenty-three months after diagnosis of breast cancer, colonoscopy was performed: an incomplete examination to the colon ascendens sampled 9 specimens for biopsy (Figs. 3 and 4). Multiple biopsies throughout the colon demonstrated adenocarcinoma with signet ring morphology, positive for etrogen and progesterone receptors supporting diagnosis of metastatic invasive lobular carcinoma of breast (Fig. 5). Immunohistochemistry shows the loss of E-cadherin expression (Fig. 6). The histological specimens from the stomach, right breast and colon and rectum were compared and the examination of hormonal receptor status in the histological specimens from the stomach was added. Based on this new information the description of histology from the stomach was upgraded from the original description of primary gastric dissociated gastric carcinoma with numerous signet ring cells to gastric metastasis from lobular breast carcinoma. Thirty-seven months after diagnosis of gastric metastasis there were no oncological changes according to control gastroscopy, mammography, chest X-ray, abdominal ultrasound and bone scan. The multiple breast cancer metastases in the colon ascendens, colon transversum, colon descendens, colon sigmoideum and rectum were assessed as inoperable. Two cycles of chemotherapy using capecitabine were administered, followed by paclitaxel weekly. Hormonal treatment was changed from tamoxifen $(20 \mathrm{mg})$ once daily to letrozole once daily, which was administered until progression 75 months after diagnosis of gastric metastasis. According to the control examinations, there were no other metastases in any other locations besides colorectal breast cancer metastases. The level of CEA was $5.32 \mu \mathrm{g} / \mathrm{l}$, CA 19-9: 24.28 U/ml, CA 72-4: <3 U/ml and CA 15-3: $31.5 \mathrm{U} / \mathrm{ml}$. Mild abdominal pain was noted, with weight loss of $2 \mathrm{~kg}$ during the previous month, but with good appetite, regular brown stools with normal consistency, and no vomiting. Thirty-eight months after diagnosis of gastric metastasis abdominal ultrasound revealed small to mild ascites without liver metastases. Colonoscopy confirmed multiple 


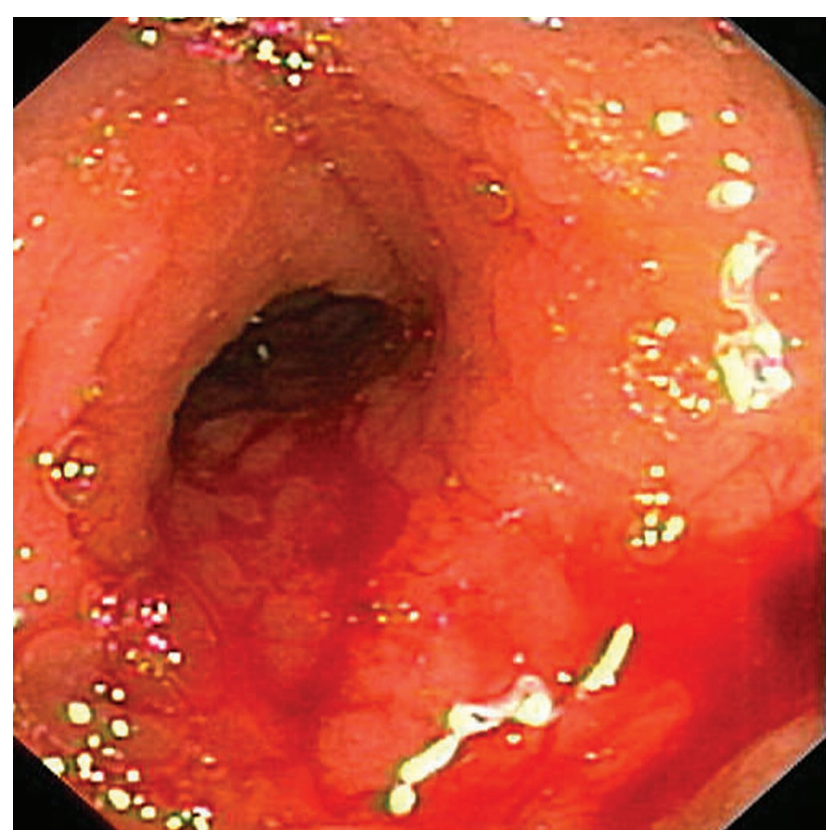

Fig. 3: Colonoscopy: Lumen of colon ascendens is narrowed; mucosa is saturated with deleted vessels. There is a bleeding spot on the righthand side of the figure. Histological examination showed large bowel mucosa focally infiltrated by the structures of dissociated adenocarcinoma.

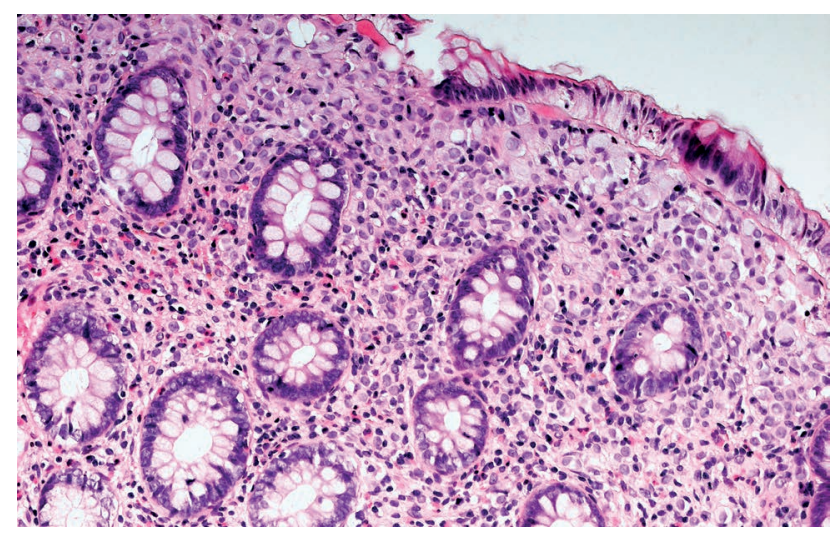

Fig. 4: Histological examination shows large bowel mucosa focally infiltrated by the structures of dissociated adenocarcinoma (hematoxylin-eosin stain).

colorectal metastases, histologically dissociated adenocarcinoma with signet ring cell morphology. The level of CEA was $7.12 \mu \mathrm{g} / \mathrm{l}$, CA 19-9: $20.22 \mathrm{U} / \mathrm{ml}$, CA 72-4: $16.09 \mathrm{U} / \mathrm{ml}$ and CA 15-3: 66.1 U/ml. Hormonal treatment changed from letrozole to fulvestrant $(250 \mathrm{mg})$ i.m. once monthly, due to progression. According to the control mammography and gastroscopy there was no local recurrence of cancer. According to scintigraphy of the skeleton there were no skeletal metastases. The performance status of the patient progressively declined: ascites, bilateral edema of the lower extremities, little bilateral pleural effusion, fatigue, weak-

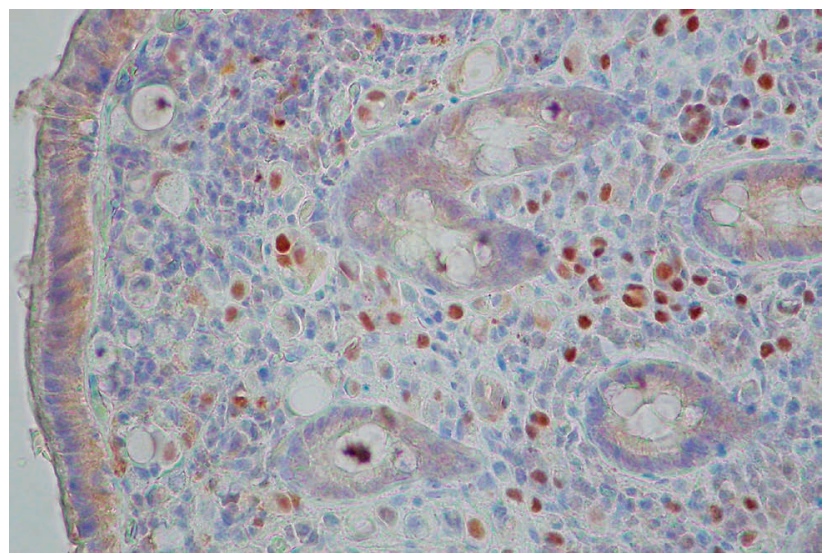

Fig. 5: Histological examination shows fragments of large bowel mucosa infiltrated by the elements of dissociated adenocarcinoma; cancer cells are positive for the expression of estrogen receptors, confirming the metastatic origin of tumor involvement of breast cancer metastases.

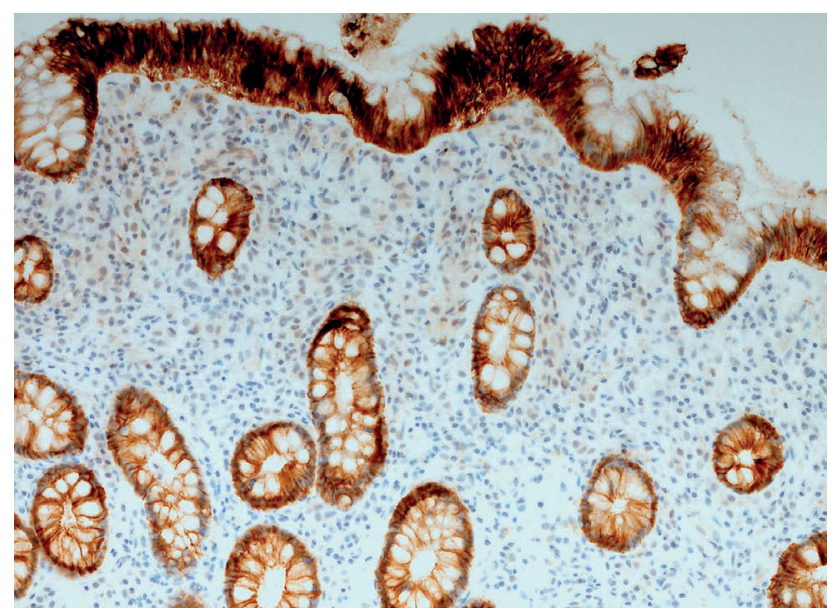

Fig. 6: Immunohistochemistry shows the loss of E-cadherin expression.

ness, lack of appetite, weight loss, flatulence and abdominal pain. Patient was treated only with hormonal treatment of fulvestrant $250 \mathrm{mg}$ i.m. once monthly; symptomatic treatment included analgesics and repeated punctures of ascites. The level of CEA was $49.89 \mu \mathrm{g} / \mathrm{l}$, CA 19-9: $187.86 \mathrm{U} / \mathrm{ml}$, CA 72-4: $153.20 \mathrm{U} / \mathrm{ml}$, and CA 15-3: $136.6 \mathrm{U} / \mathrm{ml}$. According to abdominal ultrasound there were extensive ascites, without liver metastases. Chest X-ray showed bilateral pleural effusion, more on the right side, without lung metastases. Eighty-six months after diagnosis of gastric metastasis the patient died due to progression of cancer.

\section{Discussion}

From the point of view of the clinician the occurrence of gastric metastasis as the first symptom of breast carci- 
noma with a long period of latency before presentation of the primary breast carcinoma is rare. Subsequent multiple metastases selectively to the colorectum are not so rare. From the point of view of the pathologist the first biopsy from the stomach looked like common dissociated gastric carcinoma from the signet ring cells. It did not cross our mind to search for origin from another site. The most common sites of distant metastases from breast cancer include bones, lungs, the central nervous system and the liver (1-4). The true incidence of breast cancer metastatic to the gastrointestinal tract is not known $(3,5)$. In an autopsy series, it was reported to occur in 4-25\% of patients with known disseminated breast cancer. The liver is the most common site of breast cancer metastases in the abdomen (5). The stomach is more often involved with breast cancer metastases than the colon $(3,4,6$, 7). Solitary metastases to the stomach are more common than multiple metastases. Solitary lesions are mainly located in the middle or the upper third of the stomach (8). In this case there was a solitary lesion in the dorsal wall in the region of the transition of the fundus and antrum of the stomach. Most cases in the literature of metastatic breast cancer masquerading as primary gastric carcinoma described primary breast carcinoma first and consequently gastric metastasis $(3,9,10)$. The opposite sequence - gastric metastasis first and after 14 months of latency presentation of primary breast carcinoma - makes this case unusual. The disease-free interval between primary breast cancer and gastrointestinal metastases may range from synchronous presentation to as long as 30 years (3). In this case colorectal metastasis from breast cancer occurred 23 months after diagnosis of breast cancer. In a retrospective study of 51 patients with gastric metastases of breast carcinoma median survival from detection of gastric metastases was 10 months, with a 2-year survival rate of $23 \%$ (11). In our case survival was 86 months. Although ductal carcinoma accounts for the majority of primary breast cancers, intestinal metastases are the most common lobular type $(5,6)$. Gastric metastases usually derive from lobular rather than ductal breast carcinoma (11). In this case, in accordance with the literature, the histology of the primary breast cancer was invasive lobular carcinoma. Symptoms of colorectal metastases from breast cancer are not specific. In this case subjective problems dominated: flatulence, belching, and diarrhea, without spasms, pain, or weight loss. Symptoms usually mimic those of a second primary colorectal carcinoma or inflammatory bowel disease (6). Differential diagnoses include diverticulitis, inflammatory bowel disease and ischemic colitis (4). There is no consensus for management of colorectal metastases from breast cancer due to the small number of described cases (6). Chemotherapy and hormonal therapy are usually recommended, and in the case of solitary metastasis, also surgery $(2,11)$.

\section{Conclusions}

This case report is a very rare situation. Metastases to gastrointestinal or gynaecological tracts are more likely in invasive lobular carcinoma than invasive ductal cancer. The pathologist should determine whether or not they check estrogen and progesterone receptor status not simply by signet ring cell morphology but also by consideration of clinic-pathological correlation of the patient, such as the presence of a past history of breast cancer, or the colorectal localization of poorly differentiated carcinoma, which may occur less frequently than in the stomach. Always consider possibility of invasive lobular carcinoma metastasis if patient has a past history of breast cancer, particularly invasive lobular carcinoma, even if many years before. Also consider if CT scan appearances of stomach mimic linitus plastica i.e. gastric thickening.

\section{References}

1. McLemore EC, Pockaj BA, Reynolds C, et al. Breast cancer: presentation and intervention in women with gastrointestinal metastasis and carcinomatosis. Ann Surg Oncol 2005; 12: 886-894.

2. Michalopoulos A, Papadopoulos V, Zatagias A, et al. Metastatic breast adenocarcinoma masquerading as colonic primary. Report of two cases. Tech Coloproctol 2004; 8: S135-S137.

3. Yokota T, Kunii Y, Kagami M, Yamada Y, et al. Metastatic breast carcinoma masquerading as primary colon cancer. Am J Gastroenterol 2000; 95: 3014-3015.

4. Dhar S, Kulaylat MN, Gordon K, Lali P, Doerr RJ. Solitary papillary breast carcinoma metastasis to the large bowel presenting as primary colon carcinoma: case report and review of the literature. Am Surg 2003; 69: 799-803.

5. Law WL, Chu KW. Scirrhous colonic metastasis from ductal carcinoma of the breast: report of a case. Dis Colon Rectum 2003; 46: 1424-1427.

6. Zelek L, Cottu PH, Mignot L, et al. Gastric metastasis from breast cancer: a retrospective series of 12 patients. Am J Clin Oncol 2001; 24: 363-365.

7. Critchley AC, Harvey J, Carr M, Iwuchukwu O. Synchronous gastric and colonic metastases of invasive lobular breast carcinoma: case report and review of the literature. Ann R Coll Surg Engl 2011; 93: e49-50.

8. Aurello P, D'Angelo F, Cosenza G, et al. Gastric metastasis 14 years after mastectomy for breast lobular carcinoma: case report and literature review. Am Surg 2006; 72: 456-460.

9. Jones GE, Strauss DC, Forshaw MJ, Deere H, Mahedeva U, Mason RC. Breast cancer metastasis to the stomach may mimic primary gastric cancer: report of two cases and review of literature. World J Surg Oncol 2007; 5: 75.

10. Taal BG, Peterse H, Boot H. Clinical presentation, endoscopic features, and treatment of gastric metastases from breast carcinoma. Cancer 2000; 89: 2214-2221.

11. Matsuda I, Matsubara N, Aoyama N, et al. Metastatic lobular carcinoma of the breast masquerading as a primary rectal cancer. World J Surg Oncol 2012; 10: 231.

Received: $13 / 08 / 2015$

Accepted in revised form: 08/12/2015

\section{Corresponding author:}

David Buka, M.D., Department of Oncology and Radiotherapy, Charles University, Faculty of Medicine in Hradec Králové, University Hospital, Sokolská 581, 50005 Hradec Králové, Czech Republic; email: david.buka@fnhk.cz 